\title{
The safety and efficacy of neoadjuvant programmed death 1 inhibitor therapy with surgical resection in stage IIIA non-small cell lung cancer
}

\author{
Jiangfeng Wang, Jianqiang Li, Lei Cai, Sheng Chen, Youhua Jiang \\ Department of Thoracic Surgery, Cancer Hospital of the University of the Chinese Academy of Sciences (Zhejiang Cancer Hospital), Hangzhou, \\ China \\ Contributions: (I) Conception and design: Y Jiang, J Wang; (II) Administrative support: Y Jiang, J Wang; (III) Provision of study materials or patients: \\ J Wang, J Li, L Cai, S Chen; (IV) Collection and assembly of data: J Wang, J Li, L Cai; (V) Data analysis and interpretation: J Wang, J Li, L Cai; (VI) \\ Manuscript writing: All authors; (VII) Final approval of manuscript: All authors. \\ Correspondence to: Youhua Jiang. Department of Thoracic Surgery, Cancer Hospital of the University of the Chinese Academy of Sciences (Zhejiang \\ Cancer Hospital), Hangzhou, China. Email: jiangyh@zjcc.org.cn.
}

Background: Lung cancer ranks as the most prevalent solid cancer in the world. The non-small-cell lung
cancer (NSCLC) histological subtype accounts for the largest proportion of lung cancers. Even though
neoadjuvant therapy has shown encouraging efficacy for resectable NSCLC, there is a lack of clinical data
on the treatment of stage IIIA NSCLC patients. Therefore, we carried out an evaluation of the safety and
efficacy of programmed cell death 1 (PD-1) inhibitor as an addition to neoadjuvant chemotherapy.
Methods: This prospective study involved 72 treatment-naive adult subjects with stage IIIA NSCLC
between September 2019 and July 2020 . Two circles PD- 1 inhibitor with chemotherapy (Albumin paclitaxel
$100 \mathrm{mg} / \mathrm{m}^{2} \mathrm{~d} 1,8+$ Carboplatin AUC $5 \mathrm{~d} 1$ ) were administered intravenously every 3 weeks. The patients were
operated on between 3 and 5 weeks following the second cycle. Feasibility and safety served as the primary
endpoints for this study. The rates of pathologic complete response, complete resection, response rate, and
operative and postoperative complications among the patients were also analyzed. Results: Seventy-two patients with untreated stage IIIA NSCLC were enrolled. The postoperative pathological specimens of $21(29.1 \%)$ and 47 (65.2\%) patients suggested pathologic complete response and partial remission, respectively. Neoadjuvant PD-1 inhibitor with chemotherapy had an acceptable side effect profile, and none of the subjects withdrew from the study preoperatively due to disease progression or toxicity. According to Response Evaluation Criteria in Solid Tumors (RESIST), responses evaluated by CT scan before surgery, 21 and 47 patients achieved complete response (CR) and partial response (PR), respectively, and a single patient was evaluated as stable disease (SD). There were no postoperative deaths.

Conclusions: The outcomes of PD-1 inhibitor with chemotherapy as a novel treatment for stage IIIA NSCLC in the neoadjuvant setting are satisfactory with respect to the high R0 resection rate and low toxicity profile. Prospective comparative and longer follow-up trials are needed to confirm the long-term outcomes of this novel treatment and to reach definitive conclusions.

Keywords: Neoadjuvant immunotherapy; non-small-cell lung cancer (NSCLC); programmed cell death 1 (PD-1)

Submitted Jan 14, 2021. Accepted for publication Mar 20, 2021.

doi: $10.21037 / \mathrm{atm}-21-670$

View this article at: http://dx.doi.org/10.21037/atm-21-670 


\section{Introduction}

With the deterioration of the environment, malignant tumors have become one of the biggest threats to human life in the world $(1,2)$. Among all malignant tumors, lung cancer possesses the highest mortality rate. Lung cancers fall under 2 histological divisions, small cell lung cancer (SCLC) and non-small cell lung cancer (NSCLC), with the latter comprising $80-85 \%$ of all cases, and the proportion of lung adenocarcinoma in NSCLC is increasing year by year (3). The majority of patients with NSCLC are usually diagnosed at an advanced stage, leaving little chance for surgery. With a high rate of recurrence and the survival rate at 5 years remaining poor, the prognosis for patients with lung cancer is still inadequate (4). Therapy for NSCLC has become a big problem in the clinical setting, and chemotherapy and targeted therapy are plateaued in improving the therapeutic effect and postoperative rate. It is crucial that novel methods with greater efficacy for the treatment of lung cancer are found.

Immunotherapy, as the latest cancer treatment, can activate immune cells to kill cancer cells (5). In recent years, it has become the most advanced and effective medical treatment for cancer after chemotherapy and targeted therapy. In particular, immune checkpoint therapy, such as programmed cell death 1 (PD-1)/programmed death-ligand 1 (PD-L1) inhibitors, can achieve long-term remission in $20 \%$ of patients, and even carries the possibility of producing a curative effect, thus representing revolutionary progress in the treatment of lung cancer (6). It is of great possibility using neoadjuvant pd-1 inhibitor with surgical resection to become an important part of routine therapy for lung cancer.

PD-L1 is highly expressed in NSCLC, which may increase the susceptibility of these tumors to elimination following immune checkpoint inhibition (7). So far, neoadjuvant sintilimab or atezolizumab therapy has shown encouraging efficacy in resectable NSCLC patients $(8,9)$, but there is still a lack of clinical data on the multi-target combined neoadjuvant therapy. treatment of stage IIIA NSCLC patients. We carried out a prospective pilot study to investigate treatment safety and clinical response in patients with stage IIIA NSCLC who were administered neoadjuvant PD-1 inhibitors combined with chemotherapy. We present the following article in accordance with the TREND reporting checklist (available at http://dx.doi. org/10.21037/atm-21-670).

\section{Methods}

\section{Study design}

The authors designed the present investigation as a single cohort study, which was carried out in Cancer Hospital of the University of the Chinese Academy of Sciences (Zhejiang Cancer Hospital). Enrolled subjects were given 2 cycles of PD-1 inhibitor intravenously (nivolumab $200 \mathrm{mg}$, pembrolizumab $100 \mathrm{mg}$, and camrelizumab $200 \mathrm{mg}$ ) every 3 weeks. The change in tumor size was evaluated according to the Response Evaluation Criteria in Solid Tumors (RECIST) v. 1.1. Safety and feasibility were selected as the primary endpoints of the study. Subjects were closely monitored for toxic effects of neoadjuvant therapy, for which the National Cancer Institute Common Terminology Criteria for Adverse Events v. 5.0 was adopted. The secondary and exploratory endpoint was pathological treatment response.

\section{Patients}

The key inclusion criteria for patients were as follows: (I) 20-80 years of age; (II) histologically confirmed, stage IIIA NSCLC; (III) Eastern Cooperative Oncology Group (ECOG) performance status of 0 or 1; (IV) normal organ function, and adequate pulmonary and cardiac function; (V) no metastatic cervical lymph nodes or prior cancer therapy; and (VI) written consent obtained. The following patients were excluded: immunodeficient patients; patients receiving systemic immunosuppressive treatments, or taking corticosteroids ( $>10 \mathrm{mg}$ daily prednisolone or equivalent) or other immunosuppressive drugs; and patients with an infectious disease, clinically significant concurrent cancer, or hypersensitivity to albumin paclitaxel or carboplatin drugs.

\section{Surgery}

The procedures for examining patients were the same before and after neoadjuvant therapy. Minimally invasive radical surgery, open radical surgery or sleeve resection with routine lymph node dissection were performed. All operations were performed by our center's 6 chief physicians, each of whom had experience of more than 1,000 lung cancer surgeries. Postoperative complications occurring within 90 days postoperatively, including hematological toxicities, immune pneumonia, chylothorax, 
Table 1 Characteristics of the patients

\begin{tabular}{lc}
\hline Characteristic & Outcome \\
\hline Age, yrs & 62.2 \\
Median & $42-76$ \\
Range & $66(91.7)$ \\
Male sex, No. (\%) & $60(83.3)$ \\
Smoking history, No. (\%) & \\
Clinical T stage, No. (\%) & $3(4.2)$ \\
cT1 & $20(27.8)$ \\
cT2 & $39(54.2)$ \\
cT3 & $10(13.9)$ \\
cT4 & \\
Clinical N stage, No. (\%) & $63(7.1)$ \\
N0 & $27(53.6)$ \\
N1 & $36(32.1)$ \\
N2 & $3(7.1)$ \\
N3 & \\
Pathological type, No. (\%) & \\
Small cell lung cancer & \\
Adenocarcinoma & \\
\hline
\end{tabular}

and other pulmonary complications, were documented on the case report form. The follow-up treatment regimen (PD-1 immunotherapy alone or chemotherapy combined with PD-1 immunotherapy for at least 2 cycles) was selected according to the postoperative pathology of the patient. In the first year after treatment, the patients were observed at 3-month intervals, and in the second year, they were followed up every 6 months.

\section{Study oversight}

This study is conformed to the provisions of the Declaration of Helsinki (as revised in 2013). The institutional review board of Cancer Hospital of the University of the Chinese Academy of Sciences (Zhejiang Cancer Hospital) gave ethical approval for this study. Written consent was obtained from patients. The study was designed by the author and the manuscript was written by the author. The author guarantees the accuracy and integrity of the data reported and complies with the research plan. No person other than the author has contributed to the writing of the manuscript. No companies played role in the research or reporting of this study.

\section{Statistical analysis}

The patients were subjected to continuous monitoring for side effects and adverse events (AEs), and to determine feasibility. Our hypothesis was that treatment would not be feasible if more than $25 \%$ of patients had a probability of surgical delay of $90 \%$ or higher. Furthermore, treatment was considered to be unsafe with a risk of grade 3 or 4 toxicity greater than $25 \%$. The median length of follow-up was calculated using the Kaplan-Meier method. The scope included the entire follow-up time. The Kaplan-Meier method was also used to calculate the relapse-free survival rate, from the date of surgery to recurrence or death. The $\mathrm{p}$ value was bilateral, and for analyses, 0.05 was set as the threshold for significance unless otherwise stated.

\section{Results}

\section{Patient characteristic information}

Between September 1, 2019 and July 31, 2020, we enrolled 72 patients, all of whom received 2 cycles of neoadjuvant therapy. Baseline characteristics of the enrollees are detailed in Table 1. The median age of the cohort was 62.2 years, and 60 participants $(83.3 \%)$ were current or former smokers. There were 66 cases $(91.7 \%)$ of squamous cell carcinoma, 5 cases $(6.9 \%)$ of adenocarcinoma, and $1(1.4 \%)$ case of small cell lung cancer.

\section{Safety and feasibility of neoadjuvant therapy}

All patients completed 2 cycles of PD-1 inhibitor therapy with chemotherapy. There were no cases of intolerable toxic effects or disease progression halting treatment, and no previously unreported toxic effects were documented. In most cases, toxicities were grade 1-2 (Table 2), but 1 patient suffered AEs of grade 3, and 2 patients (2.8\%) developed grade 3 rash. No grade $\geq 4 \mathrm{AEs}$ were observed. Two grade $3 \mathrm{AE}$ patients improved quickly after hormone therapy and 
Table 2 Adverse events during neoadjuvant immunochemotherapy

\begin{tabular}{lcc}
\hline $\begin{array}{l}\text { Events during } \\
\text { neoadjuvant therapy }\end{array}$ & $\begin{array}{c}\text { Grade 1-2, No. of } \\
\text { patients (\%) }\end{array}$ & $\begin{array}{c}\text { Grade 3, No. of } \\
\text { patients (\%) }\end{array}$ \\
\hline $\begin{array}{l}\text { White blood cell count } \\
\text { decreased }\end{array}$ & $11(15.3)$ & $1(1.4)$ \\
$\begin{array}{l}\text { Neutrophil count } \\
\text { decreased }\end{array}$ & $9(12.5)$ & $1(1.4)$ \\
Anemia & $8(11.1)$ & $1(1.4)$ \\
Thrombocytopenia & $5(6.9)$ & $1(1.4)$ \\
Nausea & $28(38.9)$ & $1(1.4)$ \\
Decreased appetite & $50(69.4)$ & $1(1.4)$ \\
Fever & $4(5.6)$ & $1(1.4)$ \\
Constipation & $8(11.1)$ & $1(1.4)$ \\
Fatigue & $38(52.8)$ & $1(1.4)$ \\
Alopecia & $49(68.1)$ & $1(1.4)$ \\
Rash & $18(25.0)$ & $2(2.8)$ \\
Pneumonia & $3(4.2)$ & 1 \\
Neurotoxic effects & $1(1.4)$ & 1 \\
Hypothyroidism & $1(1.4)$ & \\
Electrolyte disturbance & $1(1.4)$ & \\
\hline & & \\
\hline & &
\end{tabular}

platelet transfusion.

CT was performed for all 72 patients before surgery to evaluate the treatment effect. Twenty-one (29.1\%) patients had a complete response, 47 (65.2\%) had a partial response, $3(4.1 \%)$ had stable disease, and 1 had disease progression (Table 3).

\section{Surgery}

All 72 patients underwent surgery. Postoperative complications are summarized in Table 4. None of the participants had intraoperative complications or died in the hospital. During the postoperative period, the most common complications were pulmonary complications (16 cases, $22.2 \%)$, hematologic complications (14 cases, $19.4 \%$ ), and pleural effusion (8 cases, $11.1 \%$ ).

\section{Pathological assessment}

A median of 24 (range, 9 to 46) lymph nodes were resected from the 72 patients. A pathological complete response (pCR) was seen in the resection specimens of 21 (29.1\%) patients. Table 3 compares the pathological evaluation

Table 3 Evaluation results of 72 patients at different stages of treatment according to RESIST

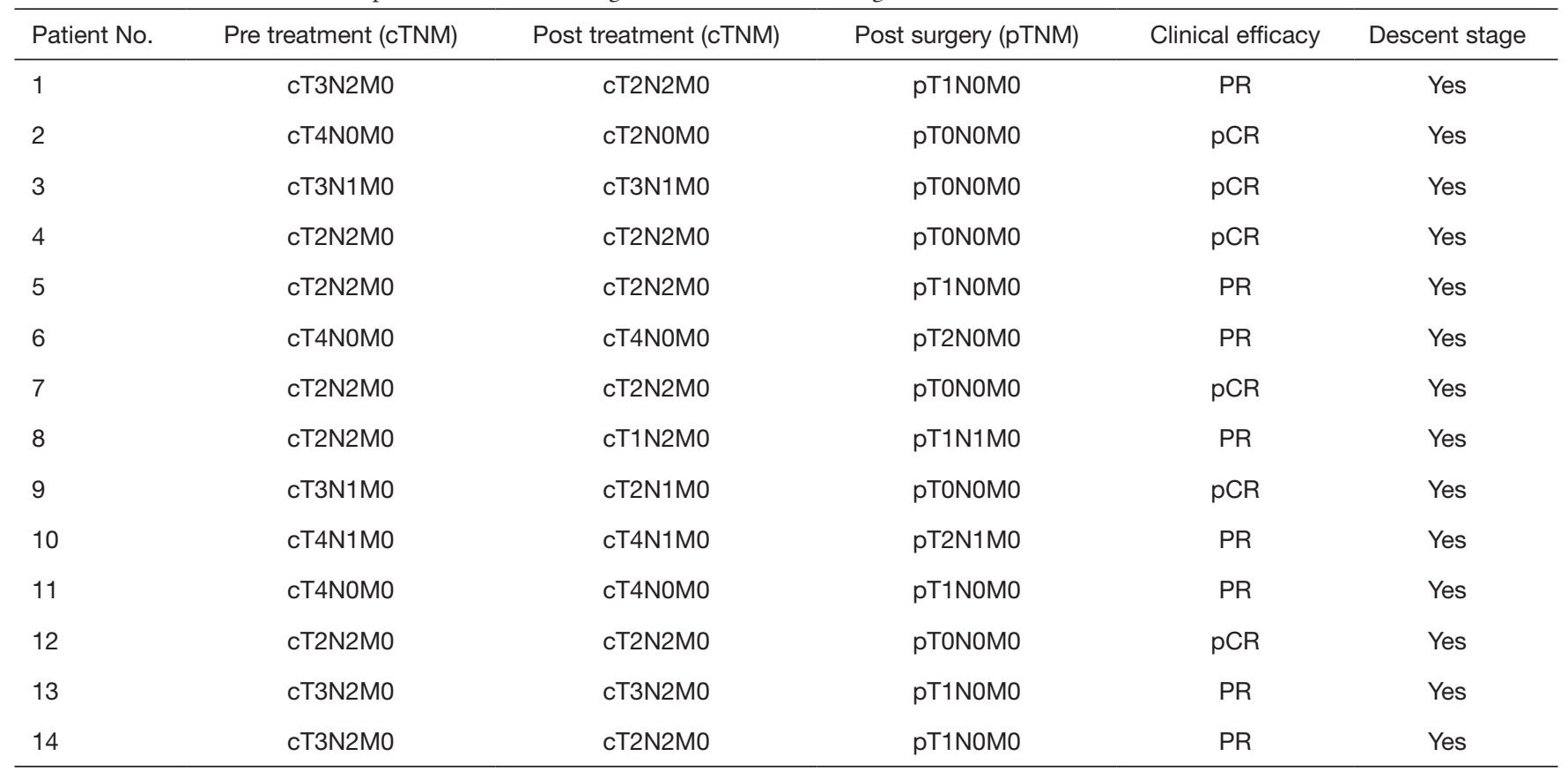

Table 3 (continued) 
Table 3 (continued)

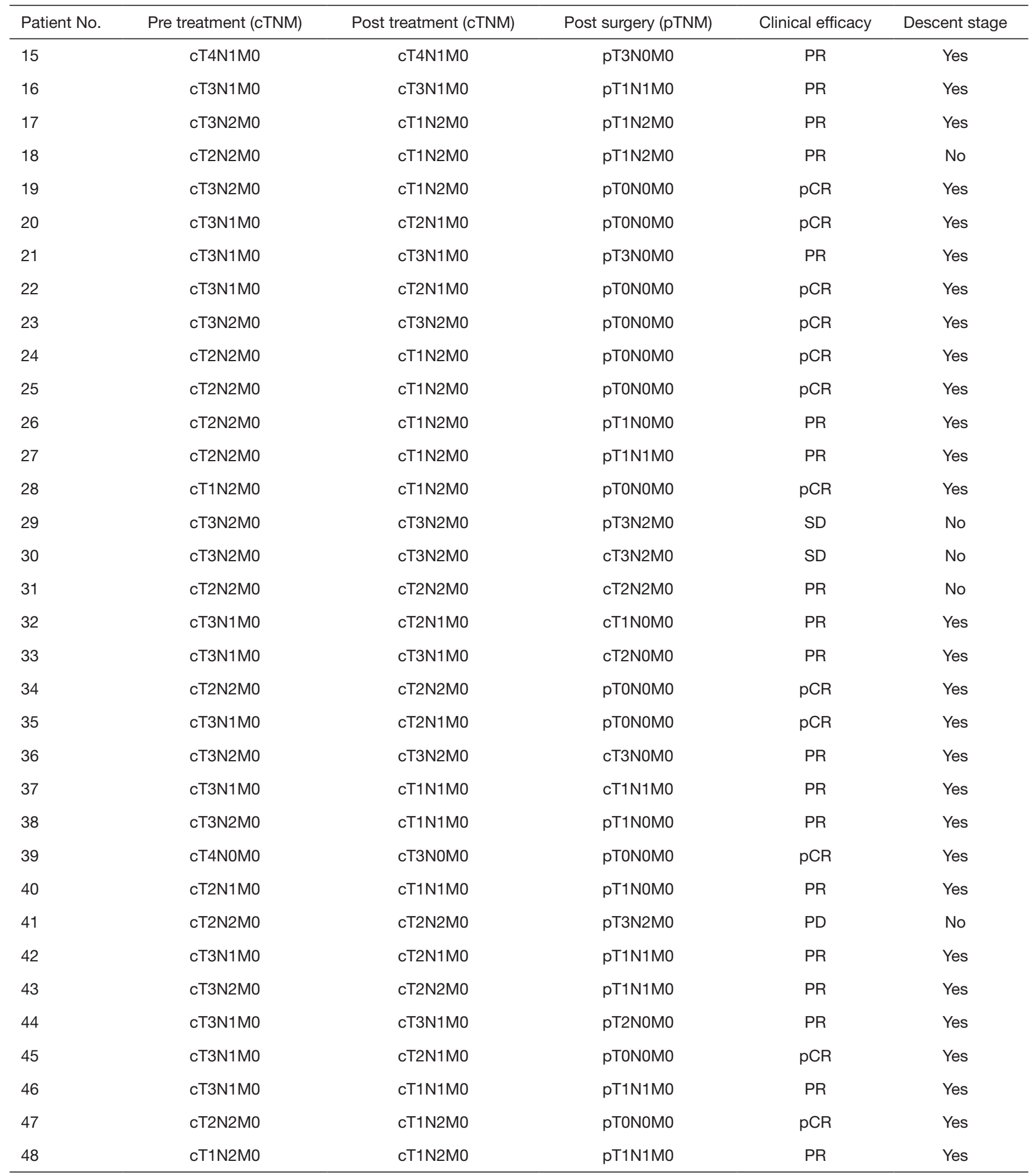

Table 3 (continued) 
Table 3 (continued)

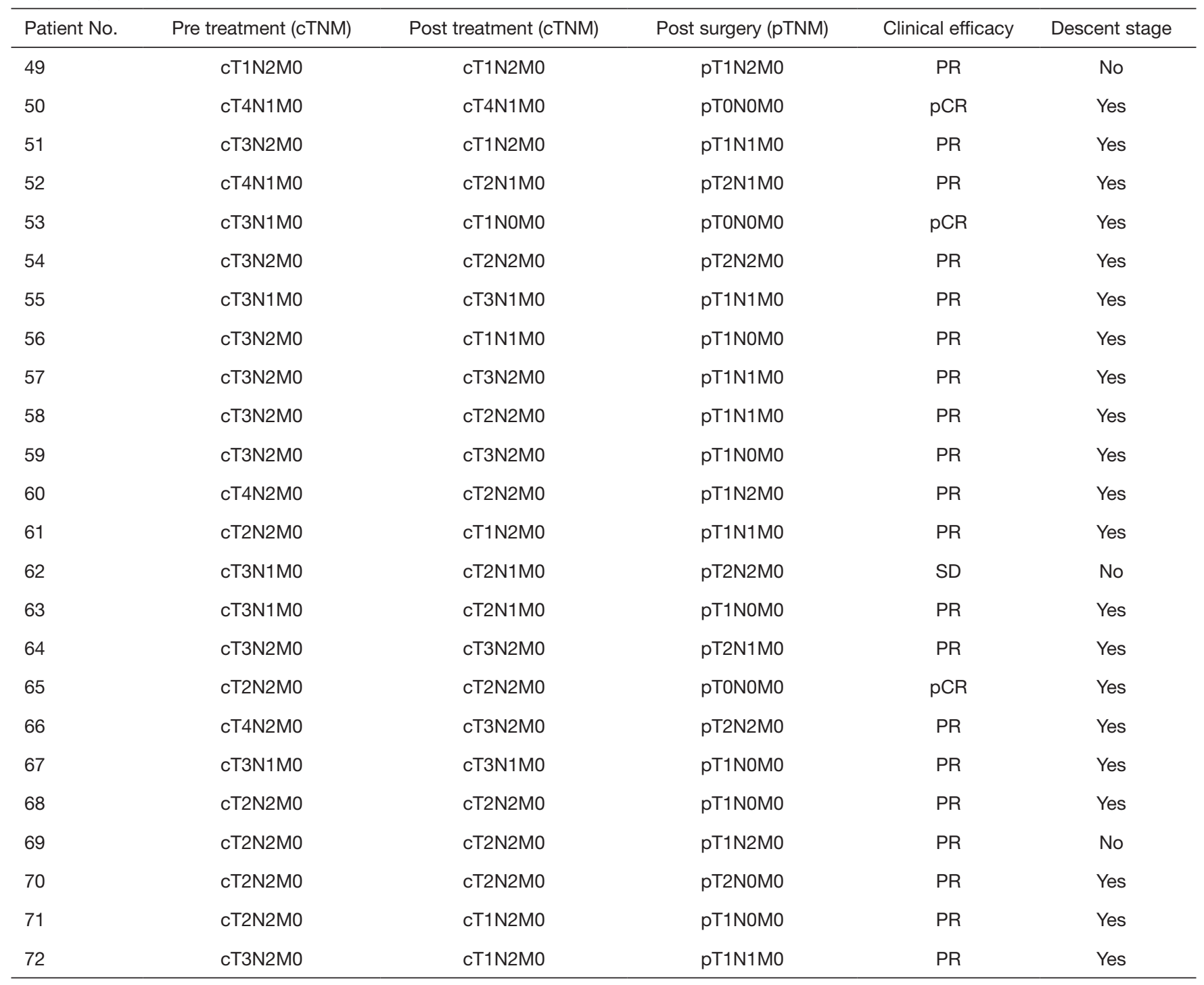

Table 4 Postoperative complications

\begin{tabular}{lc}
\hline Postoperative events & No. of patients (\%) \\
\hline Chylothorax & $3(4.2)$ \\
Pulmonary complications & $16(22.2)$ \\
Pleural effusion & $8(11.1)$ \\
Recurrent nerve paralysis & $2(7.4)$ \\
Atrial fibrillation & $5(6.9)$ \\
Pneumonia & $4(5.6)$ \\
Hematologic complications & $14(19.4)$ \\
\hline
\end{tabular}

results with the CT evaluation results for all resected specimens. Two representative specimens of pCR can be seen in Figure 1.

\section{Discussion}

Lung cancer is an aggressive disease with a poor prognosis. Surgical resection is the best option to treat early lung cancer (10). However, the early symptoms of lung cancer are not obvious, and most initial diagnoses of lung cancer are of locally advanced disease. Surgery still forms the basis of 


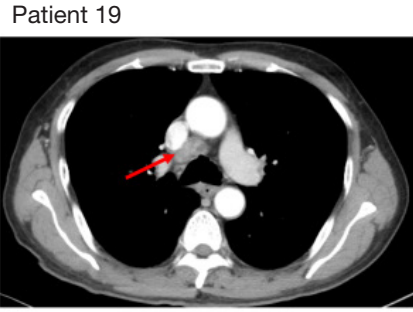

Pretreatment Imaging

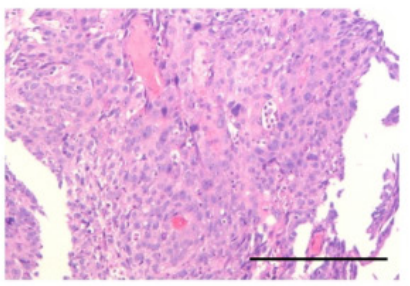

Pretreatment Tumor Biopsy

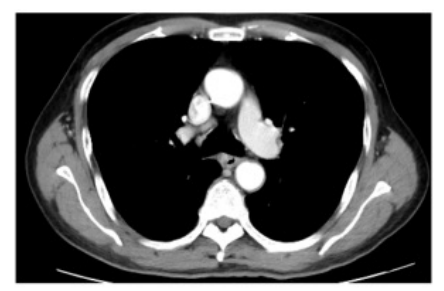

Posttreatment Imaging

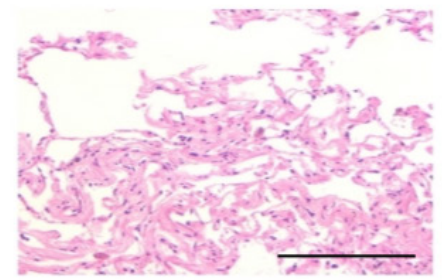

Resection Specimen

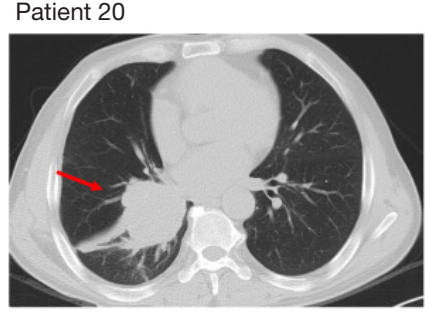

Pretreatment Imaging

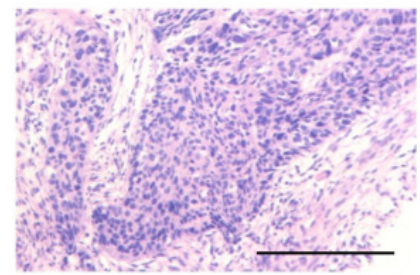

Pretreatment Tumor Biopsy

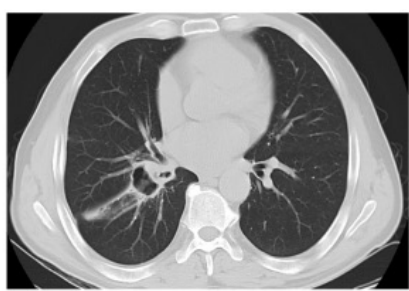

Posttreatment Imaging

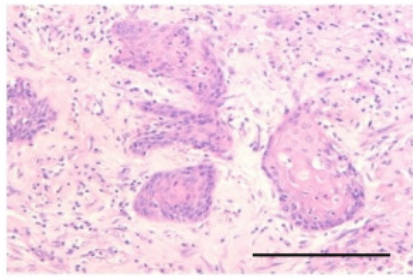

Resection Specimen

Figure $1 \mathrm{CT}$ and pathological results of 2 representative specimens of pathological complete response (hematoxylin and eosin staining). The positions of the tumor tissues are indicated by red arrowheads. Scale bar: $100 \mu \mathrm{m}$.

lung cancer treatment, but simple radical resection is often accompanied by high rates of recurrence and metastasis (11). In response to this, the treatment strategy for lung cancer has changed from the use of a single local treatment to multidisciplinary treatment, such as neoadjuvant therapy combined with surgery (12). At present, the mainstream neoadjuvant therapy for lung cancer includes chemotherapy and combined radiotherapy and chemotherapy. Neoadjuvant therapy has theoretical advantages in its control of micrometastases, which can reduce tumor staging and improve the curative resection rate (13). Many studies have shown that neoadjuvant therapy combined with surgery is significantly superior to surgery alone (14-18). However, despite NSCLC comprising the largest proportion of lung cancers, there are few reports on the efficacy of neoadjuvant treatment in stage IIIA NSCLC.

Our single-center, prospective clinical trial combined PD-1/PD-L1 inhibitor treatment with neoadjuvant chemotherapy to treat stage IIIA NSCLC before surgery. We observed a decrease in tumor grade in $60 \%$ of patients after 2 cycles of neoadjuvant therapy. The treatment was associated with few immediate AEs and no severe AEs, and there were no related cases of postponed surgery. Moreover, it achieved a pCR in $29.1 \%$ of evaluable tumors.

As mediators of negative costimulatory signals, PD-L1 and PD-1 participate in lymphocyte activation and play an irreplaceable role in immune tolerance and immune injury $(19,20)$. They are expected to become effective molecular targets for tumor immunotherapy. Tumor cells escape the surveillance and killing of the immune system by binding ligands to the receptors on $\mathrm{T}$ cell membrane. PD-L1/PD-1 inhibitors disript the interaction between receptor and ligand on tumor cells, thus blocking the signal and reactivating $\mathrm{T}$ cell-mediated anti-tumor activity. PDL1/PD-1 inhibitors, as a new therapeutic regimen for NSCLC, can improve the tumor remission rate, have low or moderate economic toxicity, and can be administered under outpatient conditions (20-23). The combination of these drugs with chemotherapy and surgery is not only suitable for elderly patients with poor organ function and a poor physical condition, but it also can be used as palliative therapy for patients who have received multi-line treatment. However, there are still some problems to be solved, such as when immune drugs should be used and for how long, and whether they should be used alone or in combination. In addition, the identification of patients who will best benefit from treatment, as well as the mechanism of drug resistance and overcoming it, still demand further research attention.

Our study was limited due to reasons including its small patient cohort and the short follow-up time after surgery. Large-cohort studies with longer follow-up and more prospective comparative trials are necessary to establish the optimal duration of neoadjuvant therapy and the best biomarkers for predicting treatment response. The correlations between pathological responses to neoadjuvant immunochemotherapy and overall survival also require 
further investigation.

\section{Acknowledgments}

Funding: None.

\section{Footnote}

Reporting Checklist: The authors have completed the TREND reporting checklist. Available at http://dx.doi. org/10.21037/atm-21-670

Data Sharing Statement: Available at http://dx.doi. org/10.21037/atm-21-670

Conflicts of Interest: All authors have completed the ICMJE uniform disclosure form (available at http://dx.doi. org/10.21037/atm-21-670). The authors have no conflicts of interest to declare.

Ethical Statement: The authors are accountable for all aspects of the work in ensuring that questions related to the accuracy or integrity of any part of the work are appropriately investigated and resolved. This study is conformed to the provisions of the Declaration of Helsinki (as revised in 2013). The institutional review board of Cancer Hospital of the University of the Chinese Academy of Sciences (Zhejiang Cancer Hospital) gave ethical approval for this study. Written consent was obtained from patients.

Open Access Statement: This is an Open Access article distributed in accordance with the Creative Commons Attribution-NonCommercial-NoDerivs 4.0 International License (CC BY-NC-ND 4.0), which permits the noncommercial replication and distribution of the article with the strict proviso that no changes or edits are made and the original work is properly cited (including links to both the formal publication through the relevant DOI and the license). See: https://creativecommons.org/licenses/by-nc-nd/4.0/.

\section{References}

1. Jemal A, Bray F, Center MM, et al. Global cancer statistics. CA Cancer J Clin 2011;61:69-90.

2. Agulló-Ortuño MT, Lopez-Rios F, Paz-Ares L. Lung cancer genomic signatures. J Thorac Oncol 2010;5:1673-91.

3. Herbst RS, Morgensztern D, Boshoff C. The biology and management of non-small cell lung cancer. Nature 2018;553:446-54.

4. Gianchecchi E, Delfino DV, Fierabracci A. Recent insights into the role of the PD-1/PD-L1 pathway in immunological tolerance and autoimmunity. Autoimmun Rev 2013;12:1091-100.

5. Franzoi MA, Romano E, Piccart M. Immunotherapy for early breast cancer: too soon, too superficial, or just right? Ann Oncol 2021;32:323-36.

6. Constantinidou A, Alifieris C, Trafalis DT. Targeting Programmed Cell Death -1 (PD-1) and Ligand (PD-L1): A new era in cancer active immunotherapy. Pharmacol Ther 2019;194:84-106.

7. Larsen TV, Hussmann D, Nielsen AL. PD-L1 and PD-L2 expression correlated genes in non-small-cell lung cancer. Cancer Commun (Lond) 2019;39:30.

8. Gao S, Li N, Gao S, et al. Neoadjuvant PD-1 inhibitor (Sintilimab) in NSCLC. J Thorac Oncol 2020;15:816-26.

9. Shu CA, Gainor JF, Awad MM, et al. Neoadjuvant atezolizumab and chemotherapy in patients with resectable non-small-cell lung cancer: an open-label, multicentre, single-arm, phase 2 trial. Lancet Oncol 2020;21:786-95.

10. Balata H, Fong KM, Hendriks LE, et al. Prevention and Early Detection for NSCLC: Advances in Thoracic Oncology 2018. J Thorac Oncol 2019;14:1513-27.

11. Osarogiagbon RU, Veronesi G, Fang W, et al. Early-Stage NSCLC: Advances in Thoracic Oncology 2018. J Thorac Oncol 2019;14:968-78.

12. Zheng Y, Jaklitsch MT, Bueno R. Neoadjuvant Therapy in Non-Small Cell Lung Cancer. Surg Oncol Clin N Am 2016;25:567-84.

13. Byrd DR, Brierley JD, Baker TP, et al. Current and future cancer staging after neoadjuvant treatment for solid tumors. CA Cancer J Clin 2021;71:140-8.

14. Stroes CI, Schokker S, Creemers A, et al. Phase II Feasibility and Biomarker Study of Neoadjuvant Trastuzumab and Pertuzumab With Chemoradiotherapy for Resectable Human Epidermal Growth Factor Receptor 2-Positive Esophageal Adenocarcinoma: TRAP Study. J Clin Oncol 2020;38:462-71.

15. Gourd E. Neoadjuvant radiotherapy improves hepatectomy survival. Lancet Oncol 2019;20:e403.

16. Wei X, Jiang Y, Zhang X, et al. Neoadjuvant ThreeDimensional Conformal Radiotherapy for Resectable Hepatocellular Carcinoma With Portal Vein Tumor Thrombus: A Randomized, Open-Label, Multicenter Controlled Study. J Clin Oncol 2019;37:2141-51.

17. Amaria RN, Menzies AM, Burton EM, et al. Neoadjuvant 
systemic therapy in melanoma: recommendations of the International Neoadjuvant Melanoma Consortium. Lancet Oncol 2019;20:e378-89.

18. Fokas E, Allgauer M, Polat B, et al. Randomized Phase II Trial of Chemoradiotherapy Plus Induction or Consolidation Chemotherapy as Total Neoadjuvant Therapy for Locally Advanced Rectal Cancer: CAO/ARO/ AIO-12. J Clin Oncol 2019;37:3212-22.

19. Kasagi S, Kawano S, Okazaki T, et al. Anti-programmed cell death 1 antibody reduces CD4+PD-1+ T cells and relieves the lupus-like nephritis of NZB/W F1 mice. J Immunol 2010;184:2337-47.

20. Dinesh RK, Hahn BH, Singh RP. PD-1, gender, and autoimmunity. Autoimmun Rev 2010;9:583-7.

Cite this article as: Wang J, Li J, Cai L, Chen S, Jiang Y. The safety and efficacy of neoadjuvant programmed death 1 inhibitor therapy with surgical resection in stage IIIA non-small cell lung cancer. Ann Transl Med 2021;9(6):486. doi: 10.21037/ atm-21-670
21. Ferrara R, Mezquita L, Texier M, et al. Hyperprogressive Disease in Patients With Advanced Non-Small Cell Lung Cancer Treated With PD-1/PD-L1 Inhibitors or With Single-Agent Chemotherapy. JAMA Oncol 2018;4:1543-52.

22. Mahoney KM, Rennert PD, Freeman GJ. Combination cancer immunotherapy and new immunomodulatory targets. Nat Rev Drug Discov 2015;14:561-84.

23. Chae YK, Arya A, Iams W, et al. Current landscape and future of dual anti-CTLA4 and PD-1/PD-L1 blockade immunotherapy in cancer; lessons learned from clinical trials with melanoma and non-small cell lung cancer (NSCLC). J Immunother Cancer 2018;6:39.

(English Language Editor: J. Reynolds) 